\title{
Resonant Cavity Enhanced Photonic Devices
}

\author{
M. Bugajski, J. Muszalski, T. Ochalski, J. Kątcki \\ AND B. MROZIEWICZ \\ Institute of Electron Technology \\ al. Lotników 32/46, 02-668 Warsaw, Poland
}

\begin{abstract}
In the present paper we review our recent works on technology, basic physics, and applications of one-dimensional photonic structures. We demonstrate spontaneous emission control in $\mathrm{In}_{x} \mathrm{Ga}_{1-x} \mathrm{As} / \mathrm{Ga}$ As planar microcavities with distributed Bragg reflectors. In general, observed trends are in agreement with theoretical predictions. We also demonstrate the operation of resonant-cavity light emitting diodes and optically pumped vertical cavity light emitting diodes developed recently at the Department of Physics and Technology of Low-Dimensional Structures of the Institute of Electron Technology.
\end{abstract}

PACS numbers: 78.30.Fs, 78.66.Fd, 78.55.-m, 78.67.De, 78.45.+h, 78.47.+p

\section{Introduction}

In recent years a number of optoelectronic devices employing microcavity structures were proposed. Such devices benefit from utilization of specific effects resulting from placing the active structure inside the Fabry-Perot type microcavity [1-4]. One of the most notable examples of such devices are resonant-cavity light emitting diodes (RC LEDs) realized in the early nineties. The main advantages of resonant cavity LEDs over conventional devices are higher emission intensities, higher spectral purity, and more directional emission patterns. All above mentioned features make RC LEDs attractive alternative for lasers in many applications. In the present paper we review our recent works on technology, basic physics, and applications of one-dimensional photonic structures. We demonstrate spontaneous emission control in $\operatorname{In}_{x} \mathrm{Ga}_{1-x} \mathrm{As} / \mathrm{GaAs}$ planar microcavities with distributed Bragg reflectors (DBRs) and report the operation of RC LEDs and optically pumped vertical cavity light emitting diodes (VCSELs) developed recently 
at the Department of Physics and Technology of Low-Dimensional Structures of the Institute of Electron Technology. The epitaxial growth issues, fabrication technology, and basic characteristics of these devices are discussed.

\section{Distributed Bragg reflectors and microcavities}

The one-dimensional photonic cavity is usually constructed normal to the substrate plane by stacking multilayer films including an active region, spacer and two dielectric mirrors. Such a structure forms a one-dimensional Fabry-Perot cavity resonator. A dielectric mirror can be formed with a periodic stack of quarter wa velength thick layers of alternating high and low refractive index material. Such a mirror is referred to as a DBR. The dielectric layers can be semiconductor layers deposited via molecular beam epitaxy (MBE). The active region consists usually of a spacer layer of the thickness equal to integer multiple of the half wavelength and of one or several quantum wells (QWs).

The simplest form of an optical cavity consists of two coplanar mirrors separated by an optical length of either one wavelength ( $\lambda$-type cavity) or one-half wavelength $(\lambda / 2$-type cavity). Coplanar cavities are the simplest form of the optical microcavities. Their properties will be discussed below. We will start with discussing properties of GaAs microcavities containing InGaAs quantum well placed in the middle of the cavity. The excitonic transitions in the quantum well are the source of spontaneous emission, which properties we want to analyze depending on the size of the cavity. The Bragg mirrors on both sides of the cavity are composed of the equal number of pairs of quarter-wavelength AlAs/GaAs layers, which makes them transparent to the radiation generated in the quantum well. What determines the spontaneous emission rate in such microcavities is the amplitude of the electric field of the standing wave of the cavity mode at the location of quantum well. In a $\lambda$-cavity quantum well is located in the antinode of the cavity, whereas in a $\lambda / 2$-cavity exciton dipole sees the node of the cavity as it is shown in Fig. 1. These two distinctively different situations should reflect in spontaneous emission properties of discussed microcavities.

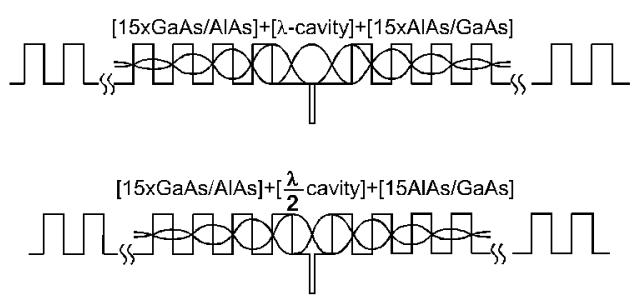

Fig. 1. Schematic illustration of the band diagram of the $\lambda$-sized and $\lambda / 2$-sized microcavities. Note the shape of the standing wave pattern in both cases. 
The optimization of the microcavity requires proper tuning of the wa velength of radiation emitted from the active region, the peak reflectivity of the DBRs, and the cavity resonance. This is the reason why the structure performance is very sensitive to the variations in thickness of the layers and their composition. The wavelength of radiation from the QW depends on both the composition and thickness. The spectral shape of the reflectivity of DBRs in the case of GaAs/AlAs reflectors depends on the layer thickness in the mirrors. Similarly, the position of the cavity resonance depends on the thickness of the spacer layers between the mirrors and the QW region and the phase of the reflection from the mirrors. Thus, the optimum performance of the structure requires simultaneous alignment of all three features.

\section{Spontaneous emission control in planar microcavities}

In this section we will discuss the effect of a planar microcavity on spontaneous emission. We will basically follow our earlier treatment of these phenomena $[5,6]$. Let us consider an elementary excitation (exciton) in solid coupled to the quantized radiation field. The coupling can be described by a perturbation term in the exciton-field Hamiltonian

$$
V=e \boldsymbol{d E} \text {. }
$$

The exciton is in initial (excited) state and drops into the final (ground) state emitting a photon of energy $\hbar \omega$. The rate of spontaneous emission $\gamma_{\mathrm{sp}}$ is given by the Fermi golden rule

$$
\gamma_{\mathrm{sp}}=\frac{2 \pi}{\hbar}|\langle i|e \boldsymbol{d} \boldsymbol{E}| f\rangle|^{2} \rho(\omega)
$$

where $e \boldsymbol{d}$ is the (vector) dipole moment, $\boldsymbol{E}$ is the electric field at the location of the exciton dipole and $\rho(\omega)$ is the density of optical modes per unit energy at the angular frequency $\omega$. It is clear from Eq. (2) that the spontaneous emission rate can be altered either by modifying the mode density, or by modifying the electric field at the location of the exciton dipole. Both the mode density and the electric field can be modified in a microcavity whose size is properly adjusted. To observe cavity related effects, field does not have to be confined in all three dimensions. One-dimensional microcavities already give sizable effects, although their strength scales with the degree of confinement. Inside the cavity, electromagnetic field forms a standing wave which meets the resonance condition, namely the round trip phase shift equal to integer multiple of $2 \pi$, as in the Fabry-Perot resonators made of metallic mirrors.

Reflectance, photoluminescence perpendicular to the Bragg reflector (PL), and photoluminescence from the edge of the structure (PL in plane) of $\lambda$-sized $\mathrm{In}_{0.2} \mathrm{Ga}_{0.8} \mathrm{As} / \mathrm{GaAs}$ planar microcavity, resonant at $1000 \mathrm{~nm}$ are shown in Fig. 2. The photoluminescence signal from the edge of the structure can be regarded as 


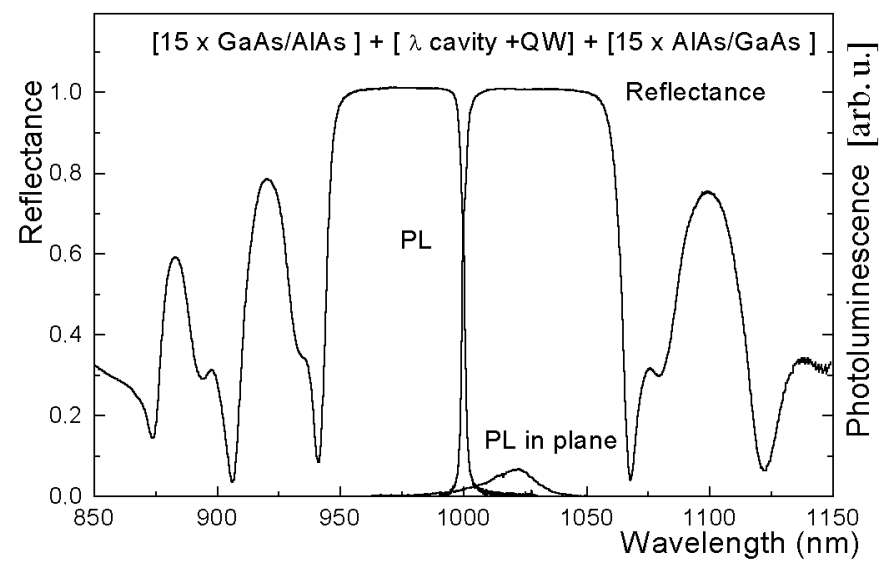

Fig. 2. Reflectance, photoluminescence perpendicular to the Bragg reflector (PL), and photoluminescence from the edge of the structure (PL in plane) of $\lambda$-sized $\mathrm{In}_{0.2} \mathrm{Ga}_{0.8} \mathrm{As} / \mathrm{GaAs}$ planar microcavity, resonant at $1000 \mathrm{~nm}$.

a reference spontaneous emission (SE) unaffected by the cavity. In the direction perpendicular to the cavity plane the $\mathrm{PL}$ signal is concentrated in a narrow line forced by cavity resonance and its intensity increases roughly by a factor of 10 (the integrated intensity of PL line increases 1.9 times). The characteristics of spontaneous emission in microcavities depend on the wavelength difference between the emitter and the cavity resonance. By lowering sample temperature it is possible to shift QW PL line to higher energies, while the position of the cavity resonance remains practically unaffected. In the case of discussed $\lambda$-sized cavity, temperature

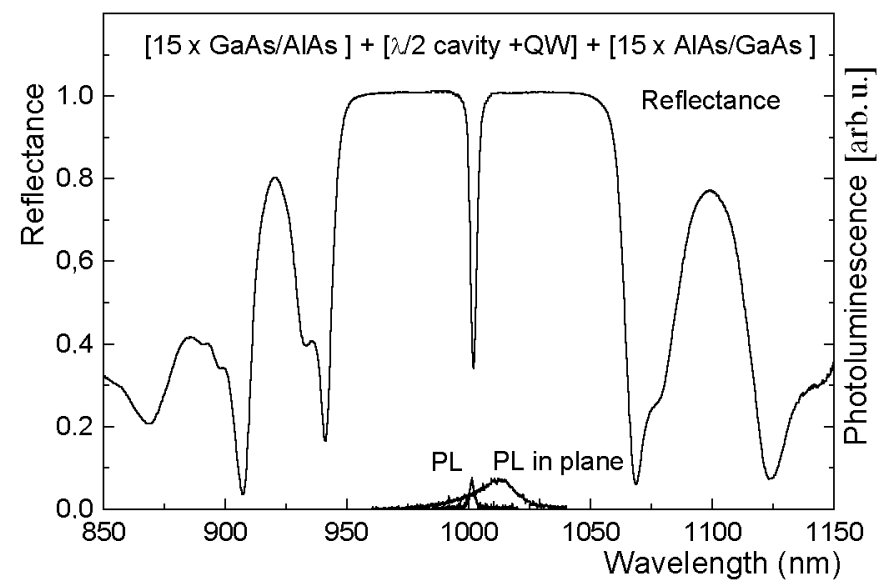

Fig. 3. Reflectance, photoluminescence perpendicular to the Bragg reflector (PL), and photoluminescence from the edge of the structure (PL in plane) of $\lambda / 2$-sized $\mathrm{In}_{0.2} \mathrm{Ga}_{0.8} \mathrm{As} / \mathrm{GaAs}$ planar microcavity, resonant at $1000 \mathrm{~nm}$. 
tuning produced further increase in PL signal by another factor of 10 , which leads to total enhancement of PL signal by the microcavity by about 100 . This is to be compared with calculated cavity enhancement factor equal to 190.

The another type of the cavity is $\lambda / 2$-sized microcavity in which QW positioned in the center of the spacer is located at the node of standing wave pattern of the cavity mode. Reflectance, photoluminescence perpendicular to the Bragg reflector (PL), and photoluminescence from the edge of the structure (PL in plane) of $\lambda / 2$-sized $\operatorname{In}_{0.2} \mathrm{Ga}_{0.8} \mathrm{As} / \mathrm{GaAs}$ planar microcavity, resonant at $1000 \mathrm{~nm}$ are shown in Fig. 3. As it might be expected this cavity effectively quenches PL signal (the ratio of integrated intensity of $\mathrm{PL}$ perpendicular to $\mathrm{PL}$ in plane signal is $1 / 12$ ). The fact that there is still some PL emitted from the cavity is due to two factors. First is that we collect PL signal from small solid angle around direction perpendicular to the cavity, second is that QW might be slightly of center with respect to intended position and consequently exciton dipole interacts with a nonzero field amplitude. Nevertheless, the PL quenching by $\lambda / 2$-sized cavity is beyond any doubt. This is an important result because it once again proves that spontaneous emission is not an inherent property of emitter but it is indeed a stimulated emission, stimulated by vacuum field fluctuations of the electromagnetic field in the cavity.

\section{Resonant cavity light emitting diodes}

The RC LED structures studied in this work were fabricated by solid source MBE. Since in the case of DBR growth the very high precision of the grown layer thickness is required, the special attention was paid to the growth rate calibration. The growth rate was calibrated by the standard reflection high-energy electron diffraction (RHEED) oscillation technique. This was done in a way to exclude the possible sources of errors. The substrate temperature during the calibration procedure was set to the $520^{\circ} \mathrm{C}$, the one used to grow the RC LED structures. In addition to the calibration done prior to the growth, the RC LED runs were controlled in situ using the pyrometric interference technique [7]. The pyrometric interference technique is a very convenient alternative to the most commonly used in situ reflectometry. Its idea is based on the simple fact that in the case of heterostructures growth the layers of different composition have different optical constants and as a result the thermal radiation generated in the substrate undergoes multiple reflections at the internal interfaces and the surface of growing structure. Consequently, the various reflected waves interfere. This interference can be observed using narrow band width pyrometer. The oscillations of the pyrometric signal are the function of the growing layer thickness. The phase and the period of oscillations provide information on the actual growth rate. The oscillations amplitude can be as high as $15^{\circ} \mathrm{C}$. Although this does not permit the instant temperature measurements, the mean value taken over a long time can be correlated with the true substrate temperature. The RC LED structures consist of two 
(a)

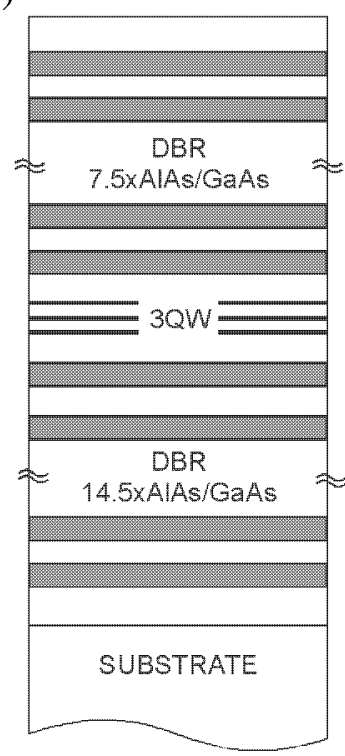

(b)
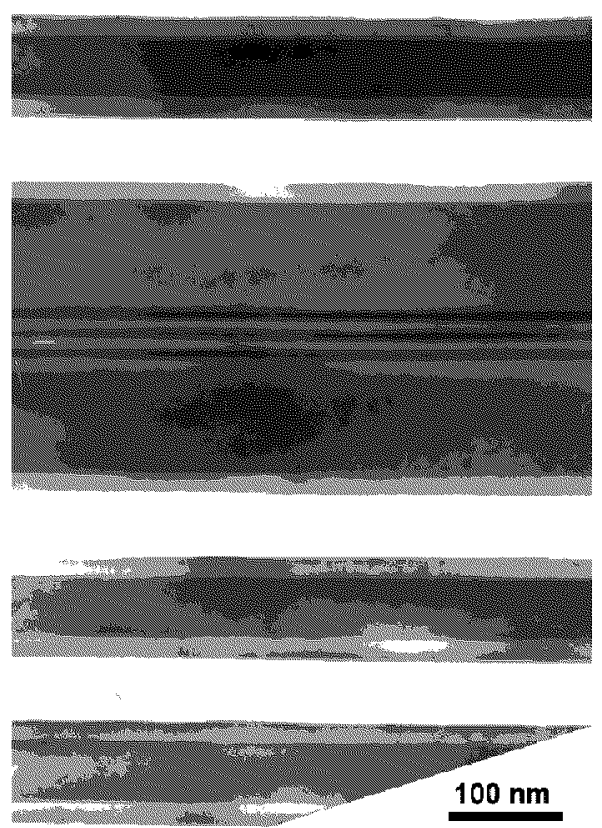

Fig. 4. (a) Schematic RC LED structure with InGaAs $3 \mathrm{QW}$ active region, designed for operation at $1000 \mathrm{~nm}$ at $T=300 \mathrm{~K}$. (b) TEM picture of RC LED structure with InGaAs $3 \mathrm{QW}$ active region and step-like AlAs/GaAs interfaces in Bragg reflectors.

Bragg reflectors, each composed of a number of AlAs/GaAs quarter wavelength layers, embedding an active region. The active region consists of $\lambda$-type cavity with either one or three InGaAs QWs, each $80 \AA$ thick, separated by $100 \AA$ GaAs barriers. The schematic view of the structure is shown in Fig. 4a, whereas Fig. 4b shows transmission electron microscopy (TEM) picture of the grown RC LED structure. To minimize series resistance of the DBR mirrors, different construction of AlAs/GaAs interfaces were applied. The structures fabricated were of abrupt GaAs/AlAs interfaces, step-like interfaces were between the GaAs and AlAs layers. $20 \mathrm{~nm}$ thick $\mathrm{Al}_{0.5} \mathrm{Ga}_{0.5} \mathrm{As}$ layer was introduced and finally the GaAs/AlAs interface was graded using digital alloy. Figure 5 shows TEM picture of different interfaces used. Different combinations of this generic structure have been tested in order to choose the optimum device design.

The MBE grown structures were tested extensively prior to the device fabrication. The most suitable techniques of characterization of RC LED structures are reflectivity and photoluminescence. The advantages of both methods lay in the fact that they are non-destructive and relatively easy to implement. The mentioned methods provide the information on the sample as a whole. In particular, they do not allow for the investigation of individual layers composing the structure. This 

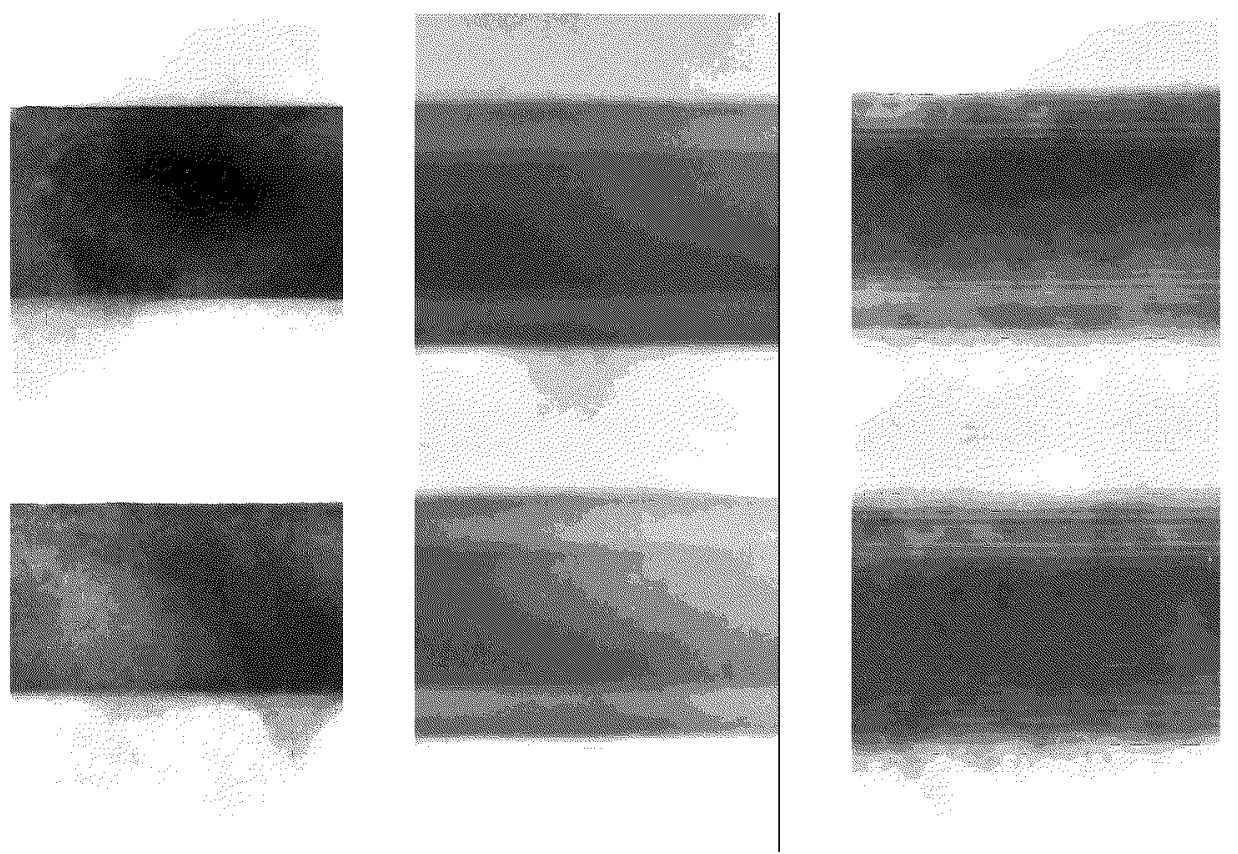

Fig. 5. TEM picture of different types of Bragg reflector interfaces used in RC LEDs.

might be a serious limitation in some cases since the RC LED can consist of as many as few hundred layers. In order to reveal the internal structure of RC LED heterostructures the TEM technique has been applied. Since it is a destructive technique it was used only for limited number of samples. On the other hand, photoluminescence and reflectivity $(\mathrm{R})$ were applied to all structures and in the first place served to qualify material for further processing. We have used high resolution PL and photoreflectivity (PR) mapping to assess spatial uniformity of the wafers with RC LED structures. The angle resolved PL mapping was used to study cavity detuning effects in the structures.

The spontaneous emission from QWs located in a microcavity is strongly modified by the cavity. The spectral width of PL line depends on the cavity finesse and can be as low as $1 \mathrm{~nm}$. This is however observed only when the PL signal is collected in the sufficiently narrow solid angle. When the light is collected in wide solid angle, the observed PL lines are substantially broadened, up to the values similar to the ones observed for the QWs without microcavity. The PL spectra measured on a microcavity structure at different directions and collected from the sufficiently narrow solid angle shift to higher energies when the angle at which the spectra are recorded, measured with respect to the normal to the sample surface, increases. This blue shift follows the relation [8]: 


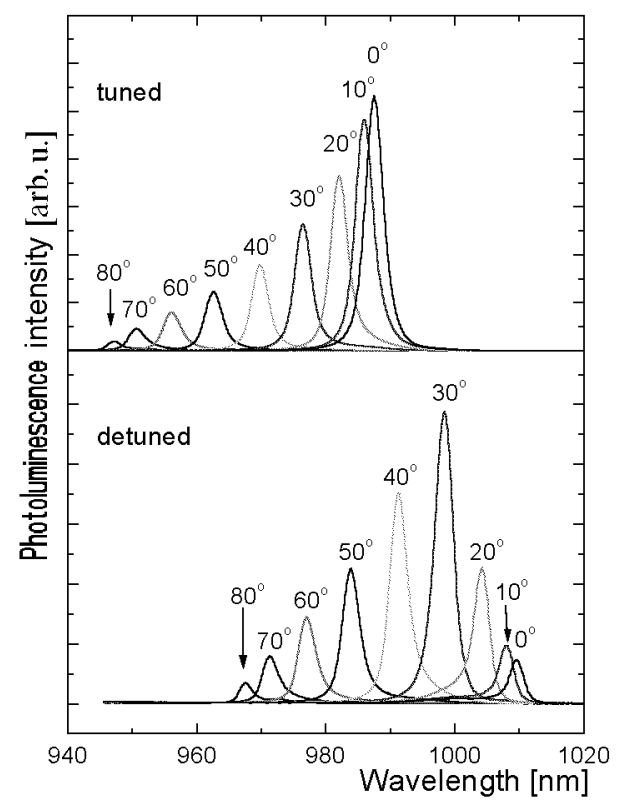

Fig. 6. PL spectra measured on RC LED structure at different directions with respect to the normal to sample surface for tuned and detuned cavity.

$$
\lambda_{(\theta)}=\frac{2 L}{m} \sqrt{n^{2}-\sin ^{2} \theta} .
$$

At the same time the emission intensity changes. The discussed features of RC LED emission are illustrated in Fig. 6 (both for tuned and detuned cavity).

The optimization of microcavity requires proper tuning of the wavelength of radiation emitted from the active region and the cavity resonance. The RC LED spectrum is determined mainly by the cavity resonance; its width decreases with the increase in the cavity finesse and the intensity increase reflect the on-axis cavity enhancement. Additional, favorable RC LED property is in this case its emission characteristic directionality. The directionality of RC LED emission also depends on the tuning between QW emission and cavity resonance. One has to keep in mind, however, that frequency of the cavity resonance depends on the angle of observation, which means that emission line shifts to shorter wavelengths with increasing angle between the direction of observation and the normal to the surface. Nevertheless, at any angle this is a narrow line in contrast to conventional LED. Summarizing, there is a very strong dependence of PL signal amplitude and wavelength on the emission direction for RC LED structures. The PL signal also depends on the QW cavity tuning. The PL spectrum width depends on the collection solid angle. Therefore, if those parameters are not properly specified, the spectral characteristics of the emission can be misleading. The total integrated spectrum of RC LED emission has been found to be actually quite broad with FWHM of the order of QW spectrum width. 
The RC LED is complicated and very demanding device, whose physics cannot be understood and consequently design and technology cannot be optimized without implementation of advanced diagnostic tools at various stages of fabrication process. The outlined approach proved to be very successful in our case and yielded commercial class devices.

The diodes were fabricated by conventional photolithography and metallization process. The light from the diode is extracted through the openings in the upper $\mathrm{Cr}-\mathrm{Pt}$ contact. The bottom $\mathrm{Au}^{-} \mathrm{Ge}-\mathrm{Ni}$ contact (to the $n$-type substrate) formed a solid circle. The diodes were electrically tested at probe tester and good ones were assembled in high frequency microwave type cases. Generally, we have found very good correlation between the results of optical tests on as grown wafers and probe tests on final devices.

The frequency of the cavity resonance depends on the angle of observation, which means that emission line of electrically pumped RC LED, similarly as it has been observed in the case of PL from the wafer with RC structure, shifts to

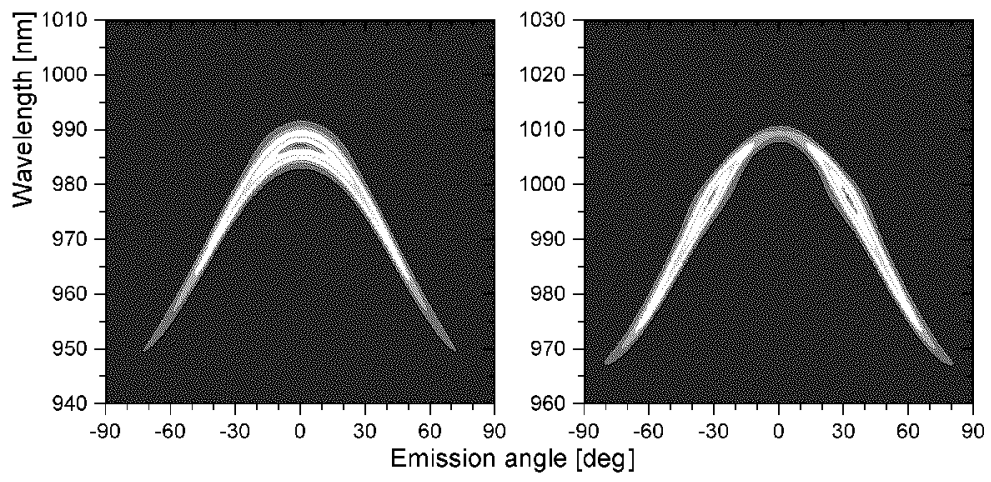

Fig. 7. 2D wavelength-angle plots of RC LED emission intensity for tuned (left part) and detuned (right part) cavity.

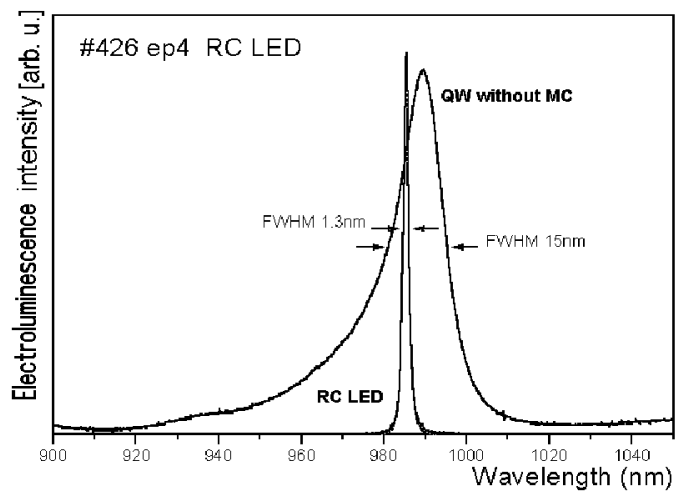

Fig. 8. Emission spectrum of RC LED compared to conventional LED. 
shorter wavelengths with increasing angle between the direction of observation and the normal to the surface. The effect is illustrated in 2D wavelength-angle plots shown in Fig. 7. Nevertheless, at any angle this is a narrow line in contrast to conventional LED which can be seen in Fig. 8. It means that we have spectral concentration in all directions. The RC LEDs can indeed be very bright. In principle, the enhancement of the spontaneous emission inside the cavity and emission through one of the mirrors outside the cavity can be very different. For very high finesse cavities, which are typical of VCSELs the overall emission out of the cavity can decrease (in the limit of very high reflectivity $R=100 \%$ the emission out of the cavity becomes zero). At moderate values of the $Q$ factor, which are characteristic of RC LEDs the spontaneous emission both inside and outside the cavity can be enhanced even by more than an order of magnitude.

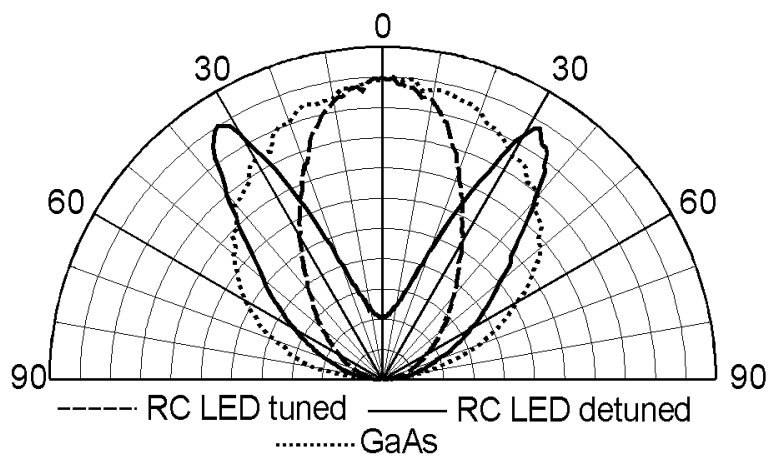

Fig. 9. Angular spectrum of RC LED compared to conventional LED.

The total wavelength integrated, intensity registered in plane of measurements differs substantially for tuned and detuned cases (Fig. 9). For comparison in the figure, there is also included the angular characteristic of conventional LED. For tuned sample we observe single lobed curve with the maximum of the signal for the direction normal to the surface, whereas the signal from detuned sample has two symmetrical maxima at wide angles corresponding to the directions at which QW emission is tuned with cavity resonance. The single lobed angular characteristic of the perfectly tuned diode shows enhanced directionality comparing to the emission of conventional LED.

\section{Vertical cavity surface emitting lasers}

Vertical cavity lasers are significantly more difficult to fabricate than their edge-emitting counterparts but the rapid evolution of their performance in recent years opened them way to many applications. The VCSEL structures discussed in this paper were grown by MBE on (100) oriented GaAs substrates using 
Riber $32 \mathrm{P}$ solid source reactor. The structures consisted of two Bragg mirrors; the lower formed by 24.5 pairs of quarter-wavelength AlAs/GaAs layers and the upper formed by 14.5 pairs of quarter-wavelength AlAs/GaAs layers. The estimated reflectivity of the lower mirror was equal to $99.7 \%$, whereas that of upper mirror was equal to $97 \%$. In between the mirrors, GaAs microcavity of the thickness $2 \lambda$, containing $3 \times 3$ InGaAs quantum wells located at the antinodes of standing wave pattern of the laser mode, was placed (see Fig. 10). The structure was intended for optical pumping experiments and as such was undoped. In real structures of electrically pumped VCSELs the Bragg reflectors, to act as carrier emitters, have to be doped to $n$ - and $p$-type conductivity going from the bottom to the top of the structure. In electrically pumped VCSEL the light is extracted through the substrate, whereas in the case of optically pumped device it emerges through the top Bragg reflector.

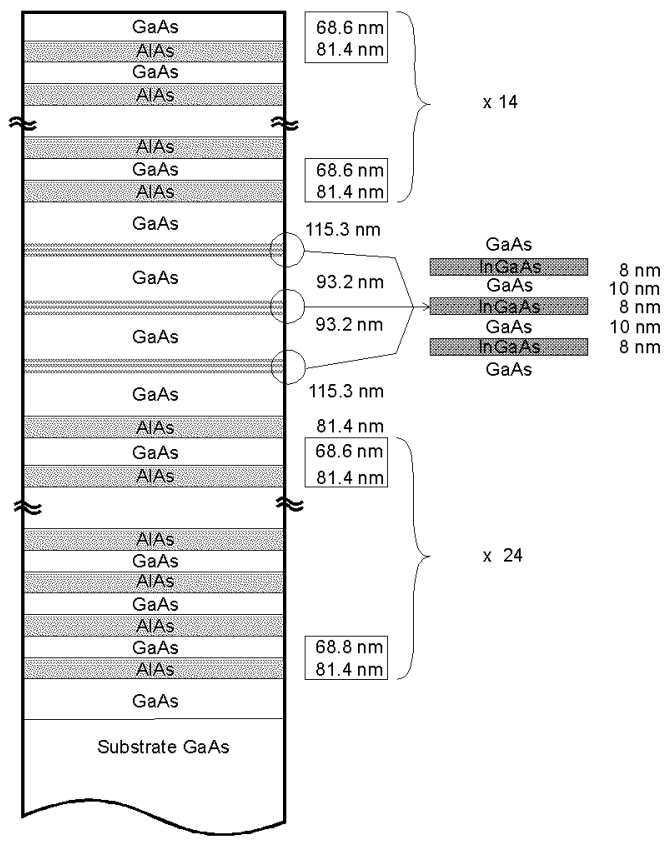

Fig. 10. Schematic VCSEL structure with $3 \times 3 \mathrm{InGaAs}$ QW active region designed for operation at $1 \mu \mathrm{m}$ at $T=300 \mathrm{~K}$.

VCSEL structures were optically excited by high power, pulse laser at room temperature. The transverse extent of the optical cavity was defined by the diameter of the laser beam and does not exceed $100 \mu \mathrm{m}$. The spectral shape and the intensity of VCSEL emission and decay of the emission as a function of pump pulse energy were measured. At low excitation densities the regular photoluminescence signal was observed. For higher excitations we have observed a clear threshold 


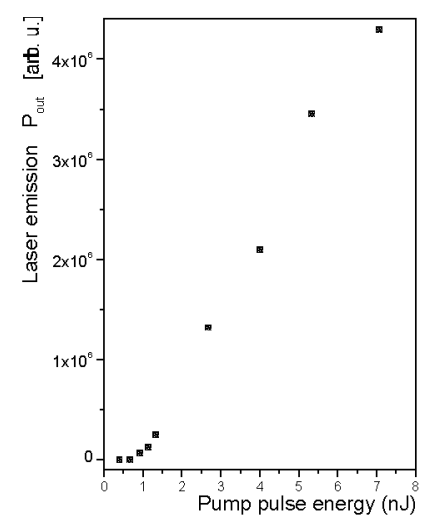

Fig. 11. Emission intensity vs. pumping pulse energy for $3 \times 3$ QW InGaAs VCSEL structure.

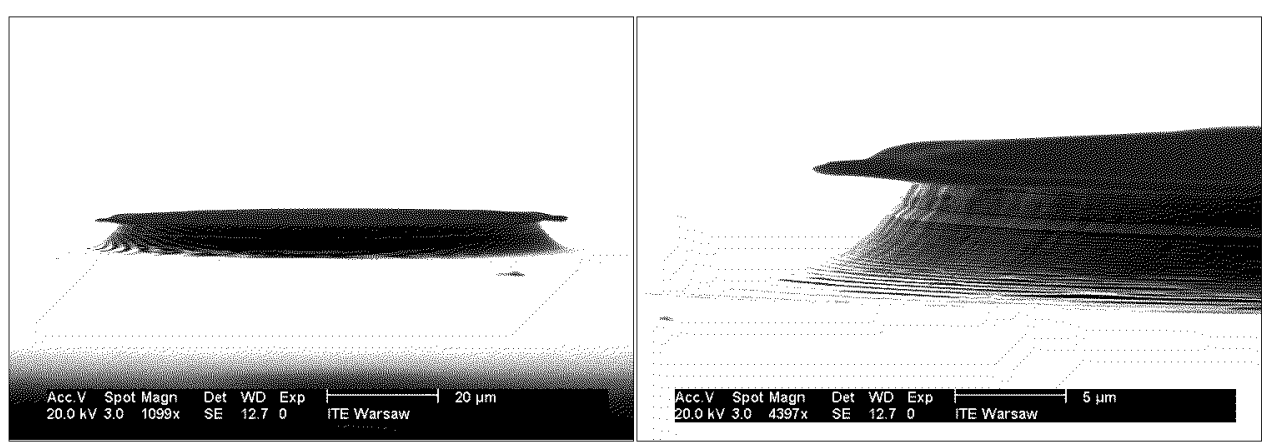

Fig. 12. SEM picture of etched-post VCSEL structures.

behavior and rapid increase in the emission intensity accompanied by the characteristic line narrowing. For very high pumping pulse energies ( $\geq 2.67 \mathrm{~nJ})$ emission starts to behave unstable and shifts to lower energies, which is the result of heating. The plot of emission intensity from VCSEL structure vs. pump pulse energy is shown in Fig. 11. The threshold occurs at pump pulse energy of about $0.7 \mathrm{~nJ}$. At the same time a marked decrease in the carrier lifetime, from about $700 \mathrm{ps}$ to $50 \mathrm{ps}$, i.e., more than one order of magnitude has been observed. The above is a behavior characteristic of the transition from spontaneous to stimulated emission, and because it coincides with other characteristic features such as threshold in the emission intensity characteristics and line narrowing, it can be regarded as a final proof of the laser action in the investigated VCSEL structures. The VCSEL structures for electrical pumping have been prepared in etched-post form (see Fig. 12) with characteristic diameters equal to $30 \mu \mathrm{m}$ and $50 \mu \mathrm{m}$. The mesa structures were prepared by wet etching. As can be seen from Fig. 12 the walls of the mesas are defect free and no lateral undercuts were produced. Also the etching depth is sufficiently controlled. 
The vertical cavity laser is in principle a zero threshold laser [9]. In conventional, edge emitting laser, only a small portion of the spontaneous emission couples into a laser mode. The rest is lost to free-space modes, which radiate in all directions. In $3 \mathrm{D}$ confined vertical cavity laser with wavelength size cavity in which only one optical mode exists whole spontaneous and stimulated emission is coupled to that mode and no clear distinction between spontaneous and stimulated regime exists. In planar $1 \mathrm{D}$ cavities due to the lack of transverse confinement there is still a threshold of laser action observed but it is substantially reduced. While in conventional lasers spontaneous emission to the laser mode coupling factor $\beta$ is of the order of $10^{-5}$ and for ideal zero threshold laser $\beta$ should equal 1 , we have obtained $\beta=10^{-3}$ for our weakly confined VCSELs; i.e., 100 times threshold reduction. Even though this is still far from theoretical limit, it is significant improvement comparing to edge emitting lasers.

\section{Conclusion}

Spontaneous emission control has been achieved in $\operatorname{In}_{x} \mathrm{Ga}_{1-x} \mathrm{As} / \mathrm{GaAs}$ planar microcavities with DBR reflectors. The room temperature emission in $\lambda$-sized cavities is enhanced comparing to its free space value while in $\lambda / 2$-sized cavities suppression of spontaneous emission is observed. The characteristics of spontaneous emission in microcavities depend on the wavelength difference between the emitter and the cavity resonance. It has been shown that ideal tuning of the cavity can be achieved by adjusting sample temperature. In general, observed trends are in agreement with theoretical predictions. These changes to the spontaneous emission process directly affect device properties. An increased coupling efficiency of spontaneous emission into the lasing mode is observed in VCSELs with $\lambda$-sized cavities. We have demonstrated reproducible MBE growth of microcavities and VCSELs.

Resonant-cavity light emitting diodes with very good emission characteristics have been also developed. RC LEDs proved to be more tolerant to the epitaxial growth parameters and device fabrication procedures. As relatively robust devices they are less sensitive to typical of VCSEL manufacturing challenges and seem to have great potential for commercialization. The problems which are still to be solved, before the technology can be regarded as mature are wafer uniformity, yield, and reliability of the devices. Nevertheless, even at the moment there is no doubt that resonant cavity enhanced devices (emitters) and VCSELs will have a profound impact on optoelectronic and photonic systems.

\section{Acknowledgments}

This work has been supported by the State Committee for Scientific Research (Poland) under contract No. 8T11B 020 18. Electron microscopy characterization 
has been supported by the State grant \#8T11B 064 19. The authors are indebted to Dr. J. Ratajczak for SEM study.

\section{References}

[1] M.S. Unlu, S. Strite, J. Appl. Phys. 78, 607 (1995).

[2] H. Benisty, H. De Neve, C. Weisbuch, IEEE J. Quantum Electron. 34, 1612 (1998).

[3] T. Baba, T. Hamano, F. Koyama, K. Iga, IEEE J. Quantum Electron. 27, 1347 (1991).

[4] C.J. Chang-Hasnain, in: Semiconductor Lasers; Past, Present, and Future, Ed. G.P. Agrawal, AIP Press, Woodbury 1995, p. 145.

[5] T.J. Ochalski, J. Muszalski, M. Zbroszczyk, J.M. Kubica, K. Regiński, J. Kątcki, M. Bugajski, in: Optical Properties of Semiconductor Nanostructures, Vol. 81 of NATO Science Series, 3. High Technology, Eds. M.L. Sadowski, M. Potemski, M. Grynberg, 2000, p. 201.

[6] K. Regiński, J. Muszalski, M. Bugajski, T. Ochalski, J.M. Kubica, M. Zbroszczyk, J. Kątcki, J. Ratajczak, Thin Solid Films 367, 290 (2000).

[7] T. Muszalski, Thin Solid Films 367, 299 (2000).

[8] P.J. Klar, G. Rowland, T.E. Sale, T.J.C. Hosea, R. Grey, Phys. Status Solidi A 170, 145 (1998).

[9] H. Yokoyama, Science 256, 66 (1992). 